\title{
MULTI-BIOMETRIC AUTHENTICATION SYSTEM USING FEATURE LEVEL FUSION
}

\author{
Anush kumar Mehalavarunan ${ }^{1}$, Sujata Kulkarni \\ ${ }^{1}$ Student, Mumbai University, India \\ ${ }^{2}$ Associate Professor, Mumbai University, India
}

\begin{abstract}
Advancements in science, technologies, industries and in numerous other fields have lead to the need for security improvements in order keep their valuables, workplace, societies, homes, etc., accessible only to authorized personnel. Biometric authentication is a well-known solution for this issue. One of the most unique patters can be obtained in finger veins which is also a unique physiological biometric. And since finger veins are beneath the skin, they are considered to be much reliable, secure and also know to be forge proof. Finger vein authentication system has proven to be a remarkable technology in the field of biometrics and are applicable where higher levels of security and privacy is very important. This paper, proposes a feature level fusion based system that uses finger vein features extracted from two algorithms namely DWT (Discreet Wavelet Transform) and canny edge detection. Results indicate better accuracy, higher TAR (True acceptance rate), lower TRR (True Reject Rate) and EER (Equal Error Rate) for feature level fusion in comparison with score level fusion technique.
\end{abstract}

Keywords_Finger vein, DWT, Edge Detection, Feature Level Fusion, Decision Level Fusion, TAR, TRR, EER

\section{INTRODUCTION}

Biometric is a part of image identification and refers to the recognition of an individual with respect to their physical and behavioral characteristics. Biometric systems area unit of 2 types' viz., physical biometry and behavior biometry. Physical biometry embrace fingerprint, hand geometries, retina, facial, etc use measurements from the body.

Table 1: Biometric Traits

\begin{tabular}{|c|c|c|c|}
\hline Biometric Trait & Authentication Method & Security Level & Privacy \\
\hline & Finger Print & Low & No \\
\hline$=$ & Retina & High & Medium \\
\hline & Knuckle & Medium & Medium \\
\hline 2 & Finger Vein & High & High \\
\hline & Palm Vein & High & High \\
\hline
\end{tabular}

Table 1 shows the security level of different biometric traits. Behavioral biometry includes keystrokes, speech, signature, etc which use dynamic measurements supported human behaviors.

\subsection{Biometric Authentication System}

Biometrics can be defined as methods to measure physical and behavioral characteristics of an individual that are used for unique recognition and verification that individual are identity. It consists of the following processes: 
- Enrollment Process

- Verification/Identification Process

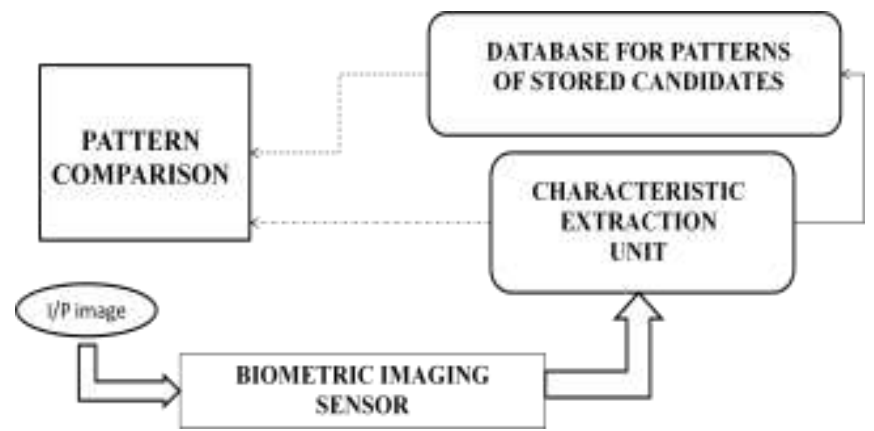

Fig 1: Block Diagram: Biometric Authentication System

Enrollment process is from input image to Database whereas the identification process includes the pattern comparison with the entire database. Figure 1 shows the the biometric authentication system's block diagram.

\section{Finger Vein}

Veins are blood carrying vessels which run throughout our body. The veins which can be found beneath the skin of a finger is know as a finger vein.

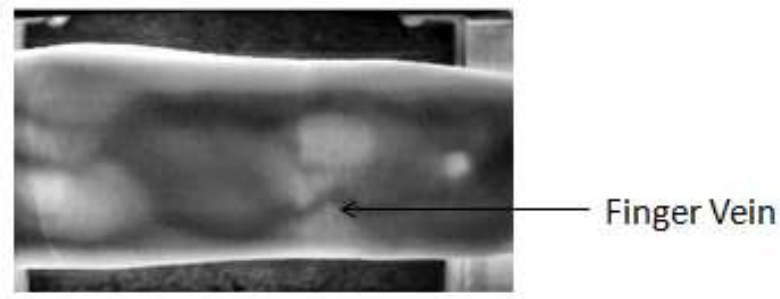

Figure 2 is a left middle finger of a person in which veins are visible. As per literature survey conducted it is proved that the finger vein recognition is more reliable and secure than the conventional modality but the error equal rate is not equal to zero[11]. These veins form a unique pattern which is different from person to person. Thus as compared to many other biometrics, finger vein are considered to be more reliable and forge proof.

\section{RELATED WORKS}

Harbi AlMahafzah [1] explained the multi-instance feature level fusion technique which was used in finger knuckle print verification. In which the experimental consequences of the combination of multiple biometrics in Feature level fusion and performance of multi-instance system were measured. Thaiyalnayaki [2] proposed an identification methodology by using 2 level discreet wavelet transform db10 which resulted in a higher compression with random additive noise. Further, mean value of each feature per individual is used for classification.

Correlation based matching approach was used in $[3,4]$ which evaluated the similarity degree of a reference subset with a different subset based on a particular criteria. Adams Wai-Kin et al. [5] came in with a feature-level-fusion technique to improve the efficiency of Palm-Code that was applied for 4 filters(Gabor) to the pre-processed palm-print images in order to compute four Palm-Codes. And Karthik Nandakumar [6] did research on the different score normalizing techniques of multi-modal biometry.

\section{PROPOSED METHODOLOGY}

Fig 2: Middle Finger

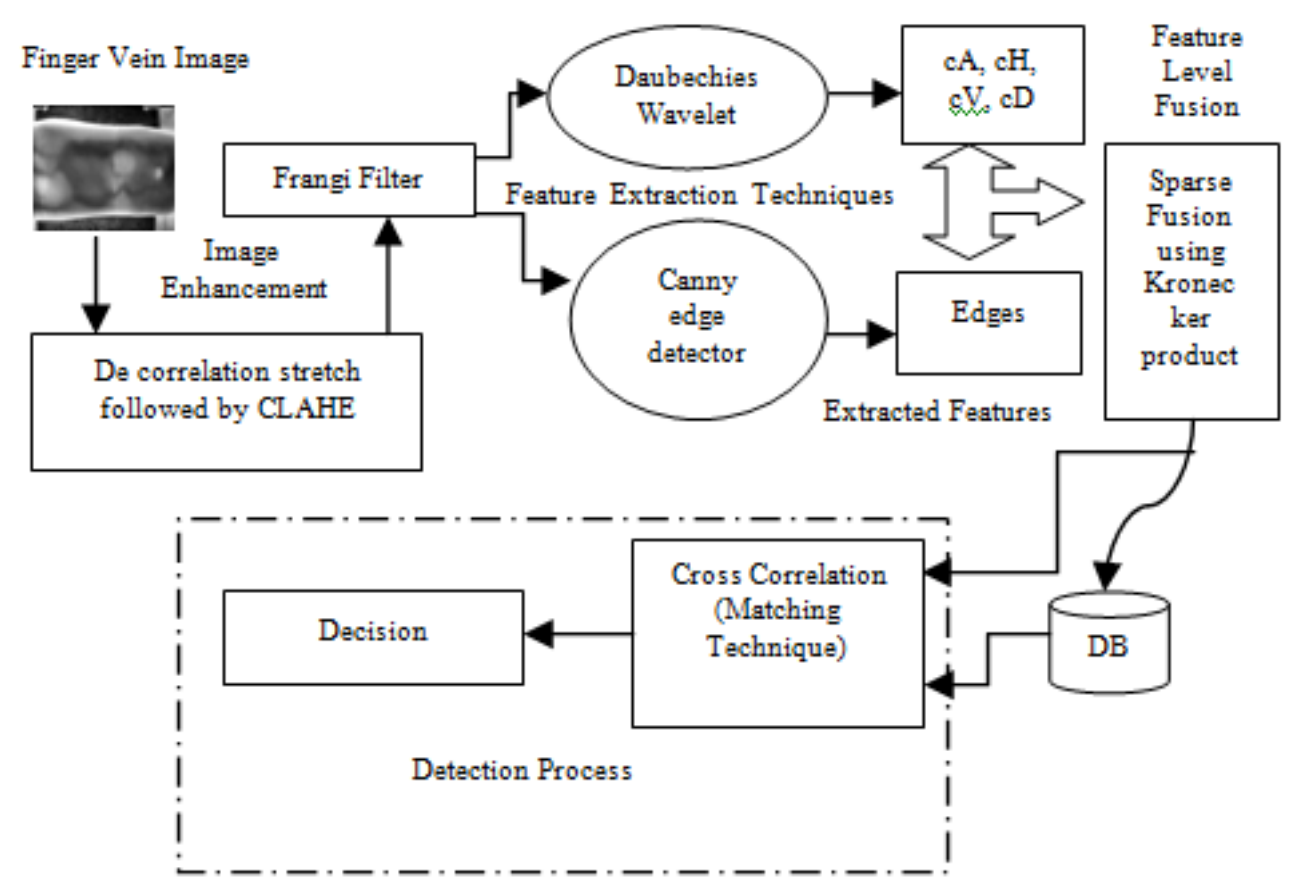

Fig 3: Proposed Block Diagram. 
Figure 3 is the block diagram of our proposed algorithm which shows the entire flow of this paper. In this paper we have used de-correlation stretch followed by Contrast Limited Adaptive Histogram-equalization method that will enhance the image and provide a more visible vein pattern. Further the frangi filter is used to detect vessel like structures and thus the unnecessary disturbances are suppresses from the enhanced image.

\subsection{Acquisition}

Generally the image is obtained from an infrared camera as the hemoglobin is in the blood in veins they do not pass through. For this paper we have obtained an open finger vein database from SDUMLA-HMT [7].

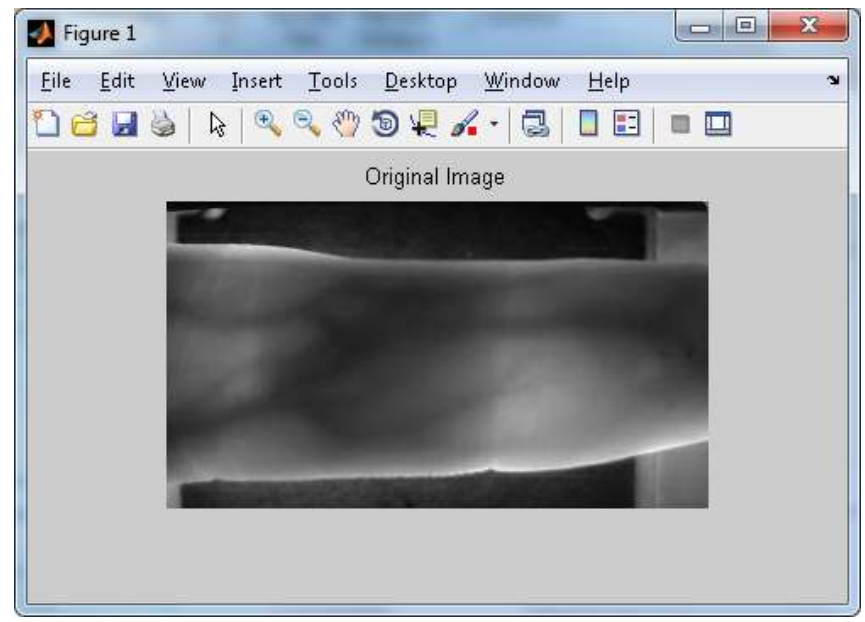

Fig 4: Sample Index finger from database

A sample index finger image is shown above in figure 4. The device used to acquire finger vein images is created for Intelligent Computing and Intelligent Systems from Wuhan university was made by the joint scientific lab. During the acquisition, every person was asked for the pictures of their ring, middle, and index fingers of each hands. Thus the assortment for the six fingers was in continual times to get 6 finger vein images. The finger vein information consists of $6 * 6 * 106=3816$ images with $320 * 240$ pixel as resolution.

\subsection{Enhancement}

Since these raw images obtained from the database do not show clear finger vein, it is mandatory to make enhance the images. In this paper we have used decorrelation stretch algorithm followed by contrast limited adaptive equalization method.

\section{1) Decorrelation Stretch:}

Decorrelation stretching enhances the separation of color in an image with significant band-to-band correlation. Decorrelation method, uses the Eigen decomposition of the band-to-band correlation matrix. In order to normalize each band by it's standard deviation . But since we have already converted the image form RGB to grayscale, only intensity enhancement takes place. We thus get a linear contrast stretched image.

\section{2) Contrast Limited Adaptive Histogram Equalization:}

Unlike histogram equalization technique, the CLAHE works on tiles for better contrast enhancements. Histogramequalization a known technique for adjusting image intensities in order to improve contrast. Let ' $\mathrm{f}$ ' be a given image represented by $(\mathrm{mr} / \mathrm{mc})$ matrix of integer-pixel intensity ranges.

In Adaptive $\mathrm{HE}$ if the area being processed has small intensity limit comparatively then the noise in this area increases considerably. The below figure will show the resultant output obtained after the CLAHE.

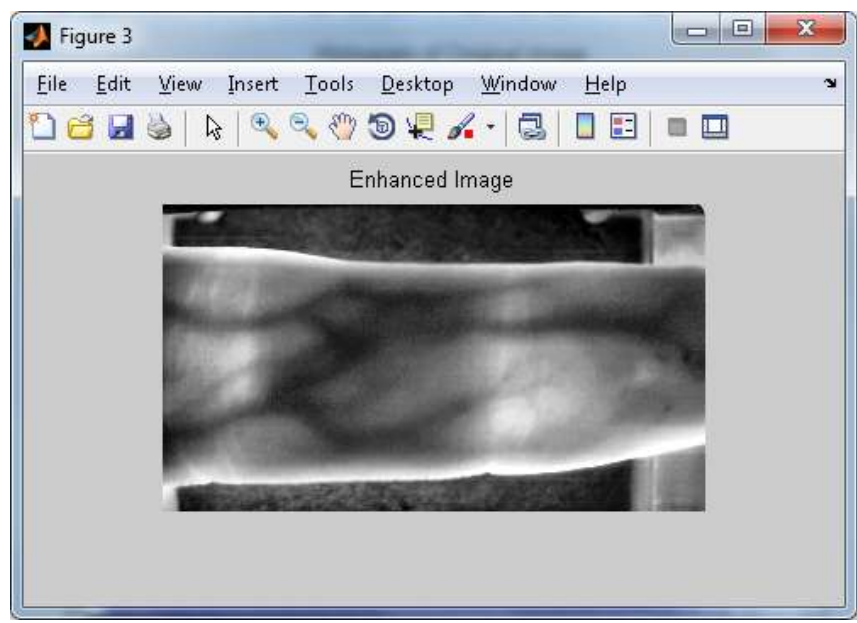

Fig 5: Enhanced image after CLAHE

Figure 5 is the output image of the contrast limited histogram equalization. It can also result to few artifact which are visible on these areas. And to restrict these artifact and noises the AHE was modified and CLAHE was made for smoother output. The total contrast enhanced for few intensities is related to slope of C.D.F function at these intensity levels. Hence, contrast can be controlled by keeping a upper cut in the slope of C.D.F. The slope of C.D.F at an area is calculated by the elevation of that histogram for that region. Therefore if range of the histogram is fixed to a certain value then the limit of the slope of the C.D.F can eventually result in the extent of contrast improvement.

$\mathrm{h}(v)=\operatorname{round}\left(\frac{\mathrm{cdf}(\mathrm{v})-\mathrm{cdfmin}}{(\mathrm{MXN})-\mathrm{cdfmin}} \times(\mathrm{L}-1)\right)$

where $\mathrm{cdf}_{\min }$ is the minimum number of non-zero values of the cumulative distribution cdf function, $\mathrm{m} \mathrm{X} \mathrm{n}$ provides us with the number of pixels and $\mathrm{L}$ is the number of grey levels being used. CLAHE followed by Bi-linear interpolation technique to remove newly added artifacts. Bilinear interpolation technique takes the weighted sum of the neighboring $2 \mathrm{X}$ 2tiles.

\subsection{Filtering: Frangi Filter}

Even after the enhancement process we can still find the noise near the vein pattern which are not needed. In order to suppress this use the filtering process. Since the veins form a vessel like structure we use frangi filter as described by 
Frangi in 2001 [8]. Frangi filter is an algorithm that uses the eigen value of the hessian matrix in order to identify curves in the image by the below formula:

$H_{i, j}=\frac{\partial_{f}^{2}}{\partial_{x_{i}} \partial_{x_{j}}}$

The axis of central vessel, $\mathrm{C}(\mathrm{v})$, can be approximated by the use of B-spline curve with a degree $n$ with $\mathrm{s}+1$ control points.

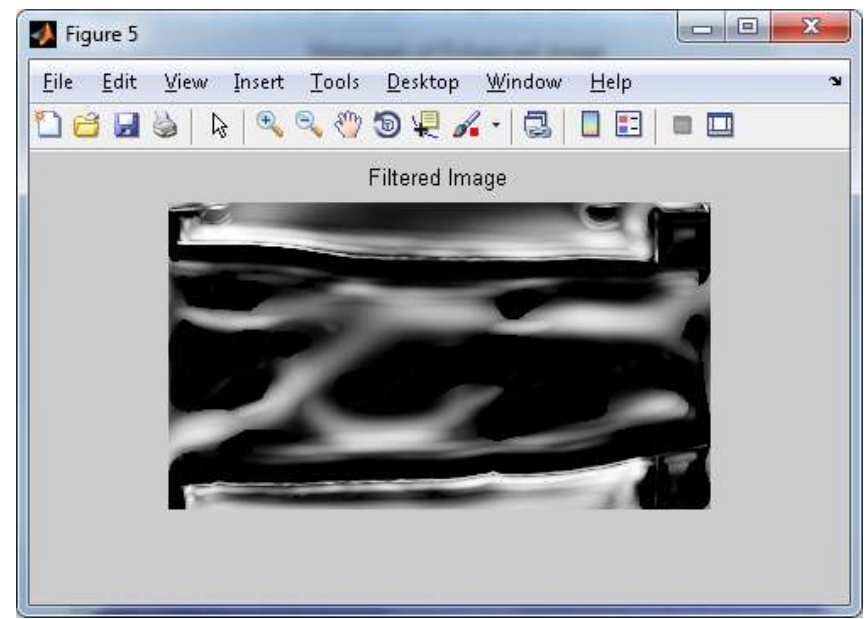

Fig 6: Frangi Filtered image

The resultant image after frangi filtering can be seen in figure 6 . Where the veins have formed as vessel like structures and the rest of the unwanted sections have been suppressed. The representation enforced the lumen line to connect:

$\mathrm{C}(v)=\sum_{i=0}^{s} \mathrm{~N}_{\text {in }}(\mathrm{v}) \mathrm{P}_{\mathrm{i}}$

Where, $\mathrm{Pi}$ is the control points; Nin(v) is the $\mathrm{i}$-th basis function of order $n$, and $v \in[0,1]$ in B-spline

\subsection{Feature Extraction}

Finger vein features can be extracted in a multi-algorithm based environment. In this paper we have extracted the Edge_feature through the Canny Edge algorithm and approximation feature through the DWT algorithm.

\section{1) Canny Edge Detection}

Canny showed that the first derivative of a Gaussian closely approximated the operator that optimize the product of localization and signal-to-noise ratio. Which is the analysis based on step-edges that are corrupted by Gaussian noise[9]. This Process of Canny edge detection is split into different steps:

Gaussian filter has been applied to smoothen the image in order for noise removal:

$$
\begin{aligned}
& H_{i, j}=\frac{1}{2 \pi \sigma^{2}} \exp \left(-\frac{(i-(k+1))^{2}+(j-(k+1))^{2}}{2 \sigma^{2}}\right) ; 1 \\
& \leq i, j \leq(2 k+1)
\end{aligned}
$$

The intensity gradients of images can be calculated by:

$$
\begin{aligned}
& G=\sqrt{G_{x}^{2}+G_{y}^{2}} \\
& \theta=a \tan ^{2}\left(G_{x}, G_{y}\right)
\end{aligned}
$$

Further non-maximum suppression is done in order to remove the spurious response of edge detection. Double threshold is applied in order to determine potential edges. This removes noise and color variation which is caused by some edge pixels. Edge tracking by hysteresis is done in order to map the edges.

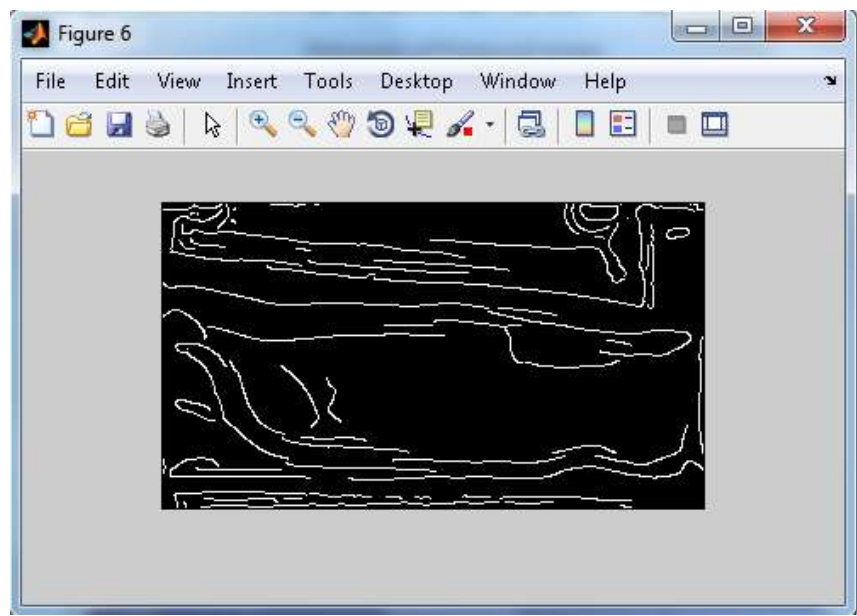

Fig 7: Edge Feature Extracted

Thus a resultant edge detected image is shown in figure 7 with only the high frequency elements which are seen in the image. These high frequency elements are stored in a matrix. Which is one of the feature which we will be using in this paper.

\section{2) Discreet Wavelet Transform}

Unlike Fourier transform, wavelet transforms provide resolution in both time and frequency. A group of wavelet function and their associated scaling functions carries out the wavelet transform in order to break down the original image into multiple sub-bands. This is repeatedly done to the sub-bands in order to produce the subsequent level.

Kekre Wavelet Transform which was tested on Finger Knuckle record resulted an enhanced recognition accuracy of $90 \%$ in comparison to usual wavelet based method obtained [12].

Daubechies mother wavelet is used in this paper:

$$
\begin{aligned}
& \quad H_{\emptyset}\left(e^{j \omega}\right)=\frac{\sqrt{2}+\sqrt{6}}{8}+\frac{3 \sqrt{2}+\sqrt{6}}{8} e^{-j \omega}+\frac{3 \sqrt{2}-\sqrt{6}}{8} e^{-2 j \omega}+ \\
& \frac{\sqrt{2}-\sqrt{6}}{8} e^{-3 j \omega}
\end{aligned}
$$


Daubechies mother wavelet is a well formed method for the construction of compact supported orthogonal wavelet. In this paper we only use one level of decomposition of $\mathrm{db} 4$ wavelet which is as shown below:

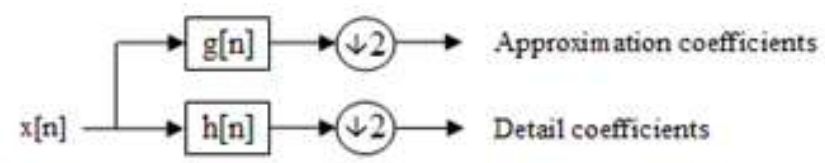

Fig 8: One level DWT decomposition

At every iteration stage of DWT the lines of the new input image which are obtained at end of the previous iteration are low-pass and high pass filtered.

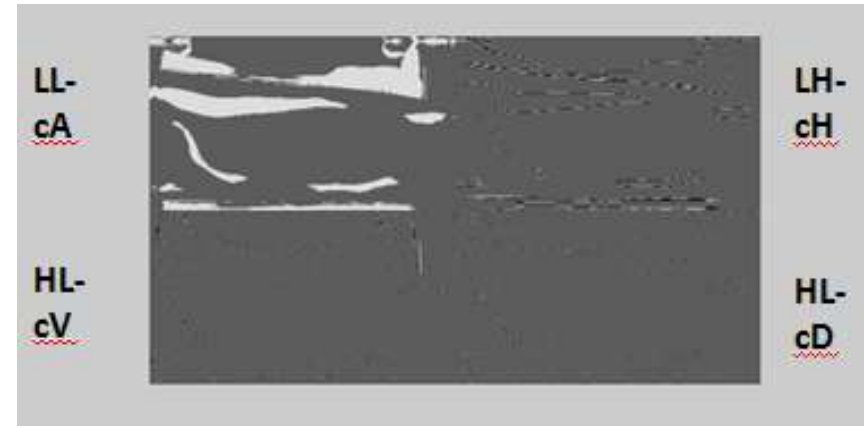

Fig 9: Output of DWT

Figure 9 Shows the output of the DWT. These lines of the two images obtained as the resultant of these H-P and L-P filters are decimated by a two. Then further the columns obtained of these two images are low passed and high passed. The columns of the 4 outputs are further made to decimate by a factor 2 . And four sub images are represented as the output for this part of the method. The image obtained after two low-pass filter is named approximation image or L-L image. The other three are named detailed sub images: $\mathrm{L}-\mathrm{H}, \mathrm{H}-\mathrm{L}$ and $\mathrm{H}-\mathrm{H}$. From which only the L-L image that is the approximation feature is taken into consideration and stored in a matrix.

\subsection{Biometric Fusion}

Fusion in biometric system, algorithms or traits are well known solutions for improving authentication of biometry systems. Now researchers have also done several experiment to prove that multi biometrics the fusion of multiple biometric features or evidences improves recognition accuracy of a system.

Levels of Fusion

- Sensor Level Fusion

- Decision Level Fusion

- Score Level Fusion

- Feature Level Fusion

\section{1) Score Level Fusion}

In score fusion the output of matching score from more than one biometric recognizers are united to find a match score which happens to be a new match-score [11,12]. In this paper for comparison we use the fused score obtained matching scores of both features by cross correlation method. The main aim of this paper is to prove that feature level fusion can produce higher accuracy and can excel in the different performance parameters as well.

\section{2) Feature Level Fusion}

In feature-fusion the extracted feature vector sets obtained through multi-algorithm techniques are united into a single unique feature by transformation, normalization, and reduction techniques operated on these features. The most significant advantage of feature-fusion is finding the related feature values made from various biometric algorithms and in this technique, identification of the salient set of features can enhance the accuracy of the recognition system. Kekre wavelet fusion technique used obtained a TAR vs TRR of $90 \%$ with EER of $10 \%$ [12]. The below figure-10 is the basic diagram of the feature level fusion that is used in this paper.

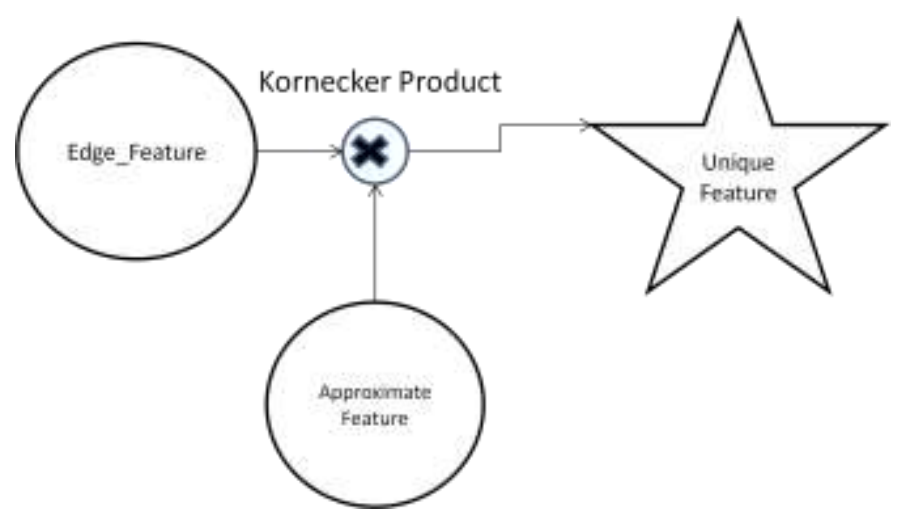

Fig 10: Block Diagram of Feature Level Fusion

a) Sparse Kornecker Product

In order to combine the two features, first the sparse matrix of both the features is formed. A sparse matrix is used for substantial memory reductions requirement can be done by storing only the non-zero values.

Consider A as the non-zero elements, JA will be the column indices and IA will be:

- The array IA is of length $m+1$. It's defined by the definition: $\mathrm{IA}[0]=0$

- $\quad \mathrm{IA}[i]=\mathrm{IA}[i-1]+$ no. of nonzero elements on the $(i-$ 1)-th row of the original matrix

Once the sparse matrix is formed both the feature vector i.e., edge feature vector and approximate feature vector and multiplied by kornecker product rule in order to form a new feature altogether as shown in figure 10.

The Kronecker product is shown by $\otimes$ and the k-product on the matrices of arbitrary size results to a (block matrix). If we have a matrix of size $m \mathrm{X} n$ and a matrix of $p \mathrm{X} q$ then the Kronecker product Matrix-1 $\otimes$ Matrix-2 is $m p \mathrm{X} n q$ (block matrix)

$(A \otimes B)_{p(r-1)+v, q(s-1)+w}=a_{r s} b_{v w}$ 


$$
(A \otimes B)=\left[\begin{array}{ccc}
a_{11} B & \cdots & a_{1 n} B \\
\vdots & \ddots & \vdots \\
a_{m 1} B & \cdots & a_{m n} B
\end{array}\right]
$$

\subsection{Matching}

Now for the identification process we use the cross correlation technique. In this technique the kornecker product of a previously trained image in database will be cross correlated with the new input image's kornecker product and will be checked with the entire database for matching.

\section{1) Cross Correlation:}

Given a matrix $\mathrm{X}$ of size $\mathrm{MX} \mathrm{N}$ and a matrix $\mathrm{H}$ of size $\mathrm{P} X$ $\mathrm{Q}$ the 2-dimensional cross-correlation, $\mathrm{C}=\mathrm{X} * \mathrm{H}$ is matrix of size $(\mathrm{M}+\mathrm{P}-1) \mathrm{X}(\mathrm{N}+\mathrm{Q}-1)$.

$$
C C F(X)=\frac{\sum_{i} P\left(X_{i}-X\right) D_{i} / \sigma_{i}^{2}}{\sum_{i} P^{2}\left(X_{i}-X\right) / \sigma_{i}^{2}}
$$

$$
\operatorname{Var}(C C F(X))=\frac{1}{\sum_{i} P^{2}\left(X_{i}-X\right) / \sigma_{i}^{2}}
$$

The above two formulas can be used for matching in between the two kornecker products. And after repeated training by trial and error a threshold value can be set.

\section{EXPERIMENTAL RESULTS}

We have trained 3 fingers viz., index, middle and ring for the left-hand of 20 subjects with 5 images of each finger. Which makes a trained database of $3 * 20=60$ fingers in total.

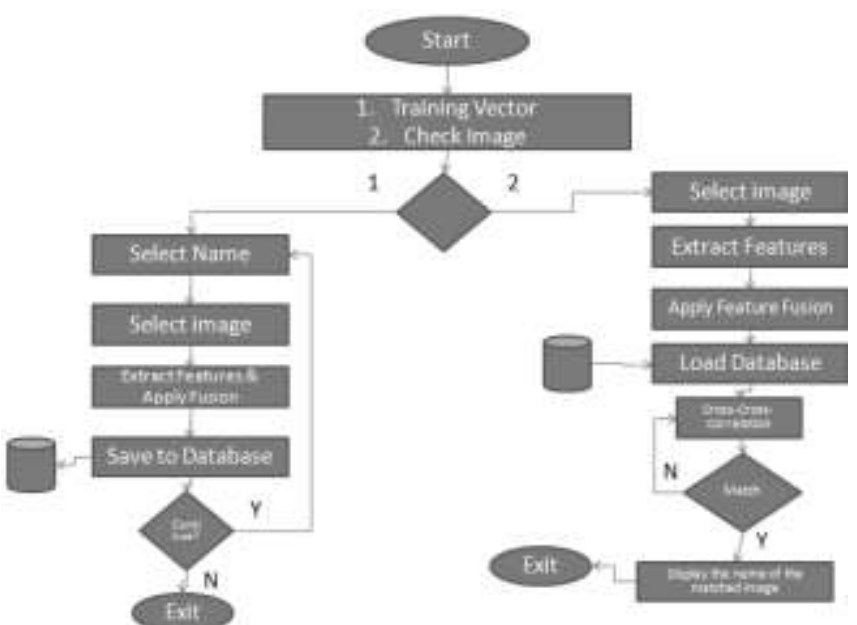

Fig 11: Flow Chart Diagram

As Shown in the above flow chart figure 11, we first input the name of the finger and the person's name and then the image's features are extracted and they are saved in the database. This is repeated for the 60 fingers for 5 times each fingers i.e., a total of 300 images in all.

\subsection{Performance Parameters}

In biometry systems, the threshold reference can be defined as the point which decides the authority to access for an individual. Authenticity can be stated for an individual who could be an intruder or genuine. The calculation of different factors such as threshold reference, False Rejection Rate, True Acceptance Rate, False Acceptance Rate, True Acceptance Rate and Recognition Accuracy are needed for a real-time biometric authentication automated system since the calculation of the biometry features are generally statistical values.

\section{1) Score Level Fusion}

The below figure 12 shows the TAR vs TRR graph which ultimately shows the recognition accuracy for score level fusion to be $81.3 \%$ only

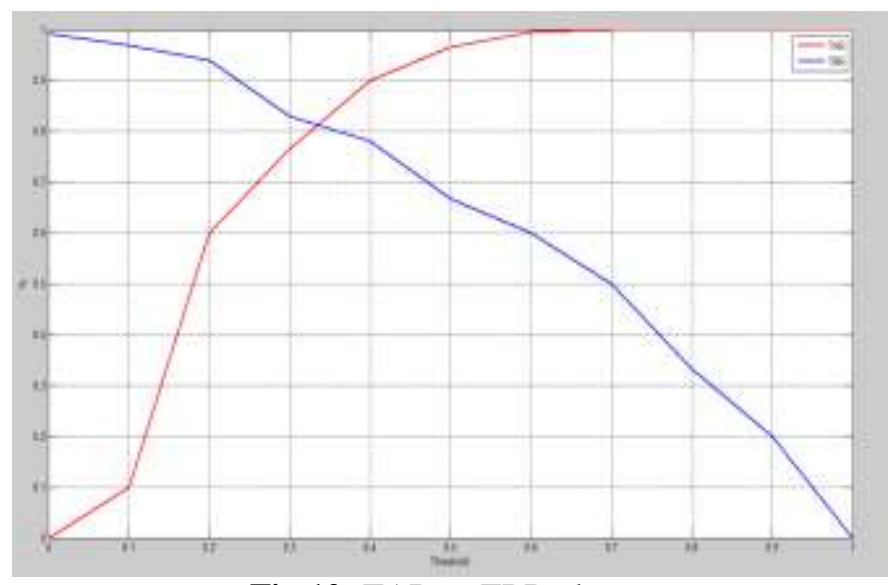

Fig 12: TAR vs TRR plot

The below figure 13 shows the plot of FAR vs FRR and the EER is noted as $19.5 \%$.

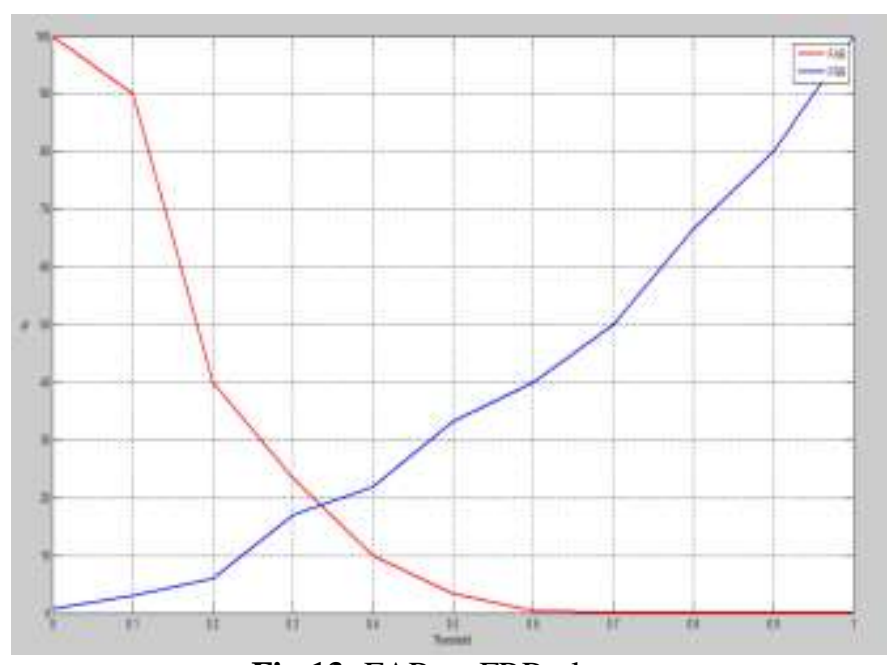

Fig 13: FAR vs FRR plot

\section{2) Future Level Fusion}

Similar to the score level fusion we plot the graph for TAR vs TRR and observe the Recognition accuracy to be $93 \%$ as shown in the figure 14 . 


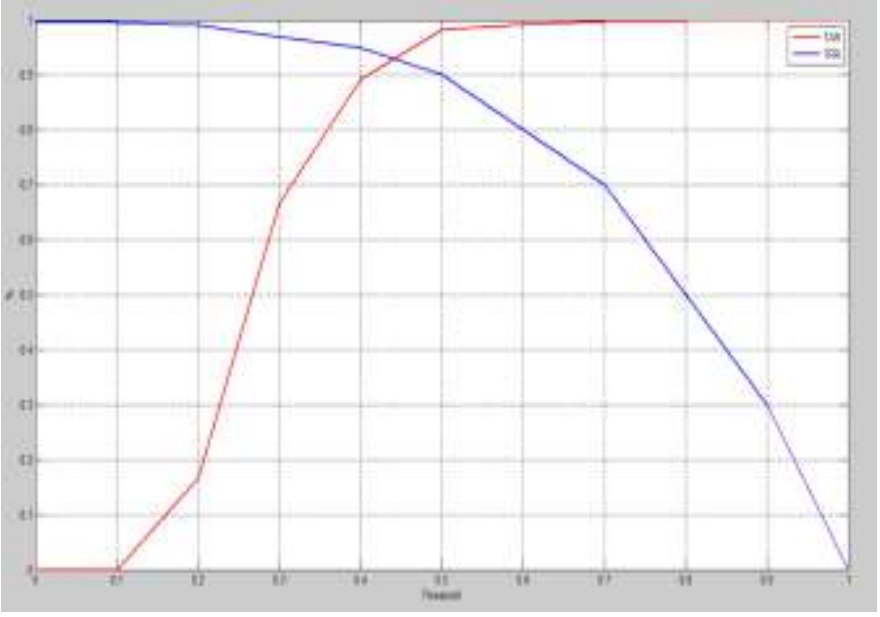

Fig 13: TAR vs TRR plot

Whereas the EER has been observed to be as low as $4 \%$ as compared to the $19.5 \%$ observed in score level fusion.

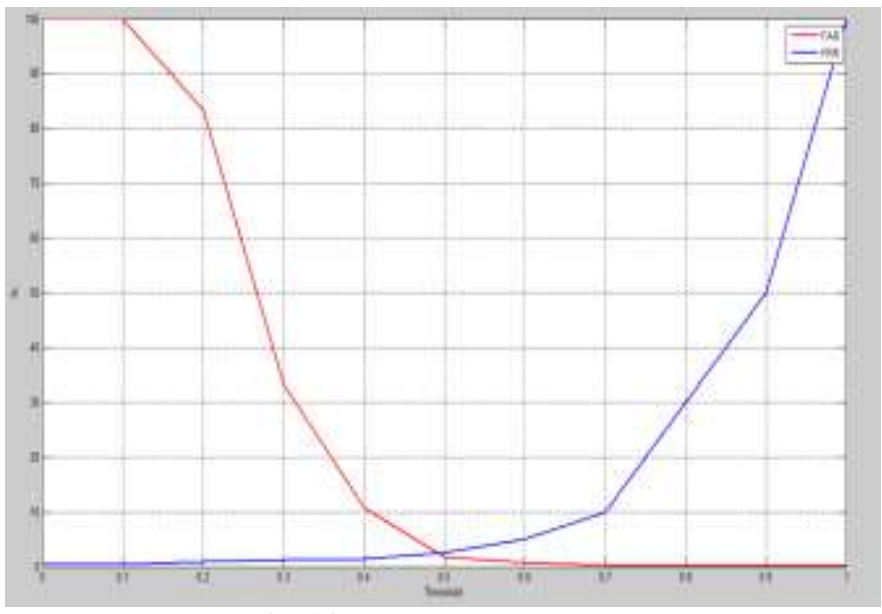

Fig 14: FAR vs FRR plot

\section{CONCLUSION AND SCOPE FOR FUTURE WORKS}

In this paper feature level fusion technique was used to combine two features into a new unique feature. As proposed the recognition accuracy of feature-fusion level was good as compared to the score-fusion level. Moreover we obtained a higher TAR and lower TRR, FAR and FRR in our proposed methodology in comparison to the scorefusion level.

Table 2: Comparison of Score vs Feature Level Fusion

\begin{tabular}{|l|l|l|}
\hline \multirow{2}{*}{$\begin{array}{l}\text { Metho } \\
\text { d }\end{array}$} & \begin{tabular}{l} 
Performance Parameters \\
\cline { 2 - 3 } \\
(TAR vs TRR)
\end{tabular} & $\begin{array}{l}\text { EER (FAR vs } \\
\text { FRR) }\end{array}$ \\
\hline $\begin{array}{l}\text { Score } \\
\text { Level }\end{array}$ & $81.3 \%$ & $19.5 \%$ \\
\hline $\begin{array}{l}\text { Featur } \\
\text { e } \\
\text { Level }\end{array}$ & $93 \%$ & $4 \%$ \\
\hline
\end{tabular}

Table 2 shows the table of comparison for score-fusion and feature-fusion technique.
Table 3: Time Complexity Comparison

\begin{tabular}{|l|l|}
\hline Method used & Time Taken \\
\hline Feature Level Fusion & 208.97 secs \\
\hline Score Level Fusion & 112.5 secs \\
\hline
\end{tabular}

Table 3 shows the time complexity comparison between score level fusion and feature level fusion technique. Thus we can conclude that though the time taken for the feature level fusion algorithm is higher than that of the score level, we get a better output as shown in table II. And in order for faster operation, we can increase the hardware configuration of the system/device by providing a dedicated hardware. Our hard work to achieve a much accurate authentication system has achieved significant results. However further efforts are required in the pre-processing stage with regards to improvement in the camera quality. The results observed in this paper has examined limitation in scale and orientation variation that are present in the dataset. Hence, more work is needed to determine the performance in a much larger environment of datasets.

\section{REFERENCES}

[1] Harbi AlMahafzah, Mohammad Imran, and H.S. Sheshadri, "Multibiometric: Feature Level Fusion Using FKP Multi-Instance biometric," IJCSI International Journal of Computer Science Issues, Vol. 9, Issue 4, No 3, July 2012.

[2] K.Thaiyalnayaki, S. Syed Abdul Karim, and P. Varsha Parmar "Finger Print Recognition using Discrete Wavelet Transform," International Journal of Computer Applications Volume 1 - No. 24, pp. 96-100, 2010.

[3] Mahri, N., Suandi, S.A.S., \& Rosdi, B.A., "Finger vein recognition algorithm using phase only correlation," IEEE Emerging Techniques and Challenges for Hand-Based Biometrics(ETCHB), International Workshop pp. 1-6, 2010.

[4] Wang, Lingyu, Graham Leedham, and David SiuYeung Cho, "Minutiae feature analysis for infrared hand vein pattern biometrics," Pattern recognition, pp. 920-929, 2008.

[5] Adams Wai-Kin Kong and David Zhang "Featurelevel Fusion for Effective Palmprint Authentication" ICBA_2004.

[6] Karthik Nandakumar, Anil K. Jain, and Arun A. Ross "Score Normalization in Multimodal Biometric Systems" Elsevier patteren Recognition, Volume 38, Issue 12, pp. 2270-2285, 2005.

[7] Yilong Yin, Lili Liu, and Xiwei Sun "SDUMLAHMT: A Multimodal Biometric Database," School of Computer Science and Technology, Shandong University, Jinan, 250101, China, 2010.

[8] A. Frangi, W. J. Niessen, and M. A. Viergever, "Threedimensional modeling for functional analysis of cardiac images," IEEE Trans. Med. Im., vol. 20, no. 1 , pp. 2-25, 2001 
[9] ] J. Canny, A Computational Approach To Edge Detection, IEEE Trans. Pattern Analysis and Machine Intelligence, Issue 8,pp:679-714, 1986.

[10] Jyoti Malik, Dhiraj Girdhar "Reference Threshold Calculation for Biometric Authentication" I.J. Image, Graphics and Signal Processing, Issue 2, 46-53, 2014.

[11] Sujata Kulkarni, Dr R.D Raut."Finger VeinRecognition," International Conference on Advances in Engineering and Technology, published by International Organization of scientific Research (IOSR), pp 32-36, 2014.

[12] Sujata Kulkarni, Dr R.D Raut, DrP.K.Dakhole "Wavelet Based Modern Finger Knuckle Authentication," Elsevier PublicationScience direct Procedia(70), pp 649-657, 2015. 